\title{
Les Antilles françaises et la départementalisation : de la domination « silencieuse » post-coloniale à l'aseptisation identitaire chez Édouard Glissant et Patrick Chamoiseau
}

El hadji CAMARA, Université Assane Seck (Sénégal)

Au moment où les Africains demandaient leurs indépendances, les Antillais privilégiaient la départementalisation présentée comme une promesse de lendemains meilleurs. En effet, si l'éveil des consciences après la deuxième guerre mondiale a conduit les intellectuels africains à revendiquer leur liberté vis-à-vis de la France, il a poussé les Antillais à faire le choix de l'assimilation politique qui s'est traduite par l'adoption puis par la promulgation d'une loi qui fait de leurs îles des Départements d'Outre-Mer (DOM) et/ou des Territoires d'Outre-Mer (TOM) dès 1946. Ainsi, les Antilles sont restées françaises, car c'était la seule issue favorable qui pourrait contribuer à la naissance d'une vraie démocratie et au relèvement du niveau de vie de leurs peuples. Sur le plan culturel également, la politique assimilationniste apparaissait comme l'unique voie capable de réunir, sous le contrôle de la France, les différentes composantes de ces sociétés dépossédées de leurs valeurs ancestrales. Pour les défenseurs de cette politique assimilationniste, devenir français pallierait la crise identitaire et rendrait la parole à ceux qui ne l'ont jamais eue. Ainsi présenté, ce tableau idyllique a emporté l'adhésion de l'élite antillaise, parmi elle Aimé Césaire qui endossera plus tard des responsabilités politiques en devenant maire de Fort-de-France puis député de la République française. Mais selon Patrick Chamoiseau et Édouard Glissant, cette politique assimilationniste demeure une forme de colonisation «silencieuse » conduisant à l'anéantissement de l'être antillais par un procédé d'aliénation culturelle insidieuse qui détruit leur(s) identité(s).

Cependant, qu'est-ce qui explique la position de ces deux écrivains par rapport à la départementalisation pourtant présentée et défendue par certains antillais, et qualifiée de «domination silencieuse » par Chamoiseau et d'acte manqué par Glissant? Quels sont les arguments contre cette départementalisation et les remèdes préconisés afin d'empêcher l'acculturation et le déni identitaire qui en résultent ? Je présenterai dans un premier temps la politique de la départementalisation telle que vécue par les Antillais dans leur majorité et dans un second temps la construction d'un contre-discours par Glissant et Chamoiseau en analysant quelques-uns de leurs essais. 


\section{La départementalisation et ses promesses d'un lendemain meilleur}

La départementalisation est un processus politique qui a consisté à accorder aux territoires d'Outre-mer le statut de département en leur attribuant des compétences qui relevaient antérieurement de l'État central français. Ainsi, la loi votée en 1946 stipulait en son article 1 que « les colonies de la Guadeloupe, de la Martinique, de la Réunion et de la Guyane française sont érigées en départements français $»^{1}$. Cette politique de départementalisation soutenue par les élites politiques de ces territoires avait entre autres buts de constituer un rempart contre les velléités d'indépendance et de résoudre les problèmes d'injustice nés du passé colonial et esclavagiste des îles.

\section{Une alternative à l'indépendance politique et à la division sociale}

En 1946, contre toute attente, les parlementaires antillais Léopold Bissol, Gaston Monnerville et Raymond Vergès ${ }^{2}$ avec à leur tête Césaire ont fait le choix historique de renoncer à l'indépendance politique de leurs îles respectives et de rester sous l'emprise de la France en portant la loi sur la départementalisation. Ainsi, malgré la violence du propos que l'on notait dans le Discours sur le colonialisme de Césaire, sorte de bilan sous forme de réquisitoire contre la politique coloniale de la France aux Antilles et en Afrique, il n'était pas question pour le poète de réclamer l'indépendance pour des raisons à la fois économiques et sécuritaires. De ce fait, la Réunion, la Guyane, la Guadeloupe et la Martinique devenant françaises, la tâche sera plus facile pour la mère patrie de les protéger contre des convoitises étrangères et d'assurer du même coup leur développement économique.

En outre, la nouvelle politique assimilationniste fait miroiter les bienfaits des lois sociales, l'augmentation du niveau de vie et le développement économique à venir. Les prestations familiales et sociales alignées sur celles de la Métropole, le développement de la politique de migration, les aides à l'industrialisation, entre autres, devaient faire taire les velléités autonomistes. C'est la raison pour laquelle toute lutte politique aux Antilles réclamant une quelconque autonomie était de prime abord vouée à l'échec. Pour preuve, l'audience des indépendantistes restera très limitée dans les îles, surtout à partir de l'année 1982, date à laquelle les départements d'outre-mer

bénéficièrent des lois de décentralisation et de régionalisation. À partir de ce moment, bon nombre de militants nationalistes feront le choix de participer à la gestion des affaires du département. Ce qui fait que la résistance et la valorisation de soi devinrent impossibles face aux valeurs coloniales dominatrices. 
C'était aussi la seule issue favorable qui pourrait contribuer à la naissance d'une vraie démocratie aux Antilles, comparée à ce qui se passait à la même époque en Afrique. Ainsi, assimiler les Antilles à la France entrainerait plus de justice sociale et permettrait d'assainir le système économique et politique de ces territoires d'outre-mer. De ce fait, la départementalisation avait suscité l'espoir de mettre fin aux inégalités sociales et de faciliter l'intégration des classes populaires défavorisées avec l'importation de biens consommables par des containers venant de la Métropole.

Ainsi, la départementalisation apparaissant comme une promesse de lendemains qui chantent, il fallait s'assimiler, c'est-à dire être français en adoptant sa culture et ses modes de vie. Du point de vue du gouvernement français, cette loi permettra d'éviter les conflits futurs entre les anciens maitres esclavagistes et ceux qui étaient à leur service. La politique assimilationniste apparaissait donc comme l'unique voie capable de réunir les différentes composantes de cette société sous le contrôle de la France. Devenir français pallierait le manque identitaire et rendrait la parole à ceux qui ne l'ont jamais eue. D'une certaine manière, c'était pour les Antillais le moyen de devenir des hommes à part entière en se hissant à la hauteur de l'ancien maître. La Départementalisation était aussi la garantie de l'égalité civile et juridique. Cependant, cette reconnaissance de l'égalité aux yeux de la loi entre ces anciennes colonies et la Métropole ne s'est jamais traduite dans les faits selon certains intellectuels antillais.

\section{La dénonciation de la politique d'assimilation à marche forcée}

La départementalisation, malgré les avancées notables sur le plan politique et l'amélioration des conditions de vie des classes sociales défavorisées qu'elle créa, est toujours vue par une certaine élite politique et culturelle antillaise comme étant la source de toutes les dépendances. Pour certains intellectuels antillais tels que Glissant, Chamoiseau et Raphaël Confiant, la départementalisation n'est autre qu'une autre forme de domination qui perpétue l'emprise de la France sur les territoires d'Outre-mer.

Dans Écrire en pays dominé, Chamoiseau dénonce le fait que Césaire et ses collègues parlementaires aient naïvement cru à la politique de la départementalisation comme étant une solution aux problèmes d'injustice nés de plusieurs siècles d'esclavage et de domination coloniale. Car pour lui, si l'assimilation politique et économique permet une amélioration des conditions de vie de la population, cela signifie aussi la perte du pouvoir de décision sur la réalité économique, culturelle et politique de ses territoires. En instaurant un système d'aide permanent sans un 
véritable plan de développement des îles, la départementalisation maintient le peuple dans une totale dépendance. Leur intégration économique a renforcé leur dépendance envers la Métropole. Jadis productrices, les Antilles sont devenues des consommatrices passives de produits occidentaux importés. À cela, il faut ajouter la soumission culturelle et sociale due au fait que les dominants finissent toujours par imposer leur perception du monde aux dominés.

Selon Chamoiseau, la politique assimilationniste reste une forme de colonisation plus cruelle, laquelle a conduit à l'anéantissement de l'être antillais par un procédé d'aliénation culturelle. La prétendue universalité des valeurs n'est qu'un moyen de promotion de la culture française dominante au détriment de celle des Antilles. Il déclare à ce propos : «La départementalisation nous sépara de nous-mêmes. Le soutien au 'Développement' (mot-culte) suscita l'extinction des esprits autonomes » (227) et l'Antillais devient stérile devant ses propres valeurs en contribuant lui-même à promouvoir la culture occidentale, et en ignorant ses valeurs de culture héritées du monde des plantations. Et comme le constate Chamoiseau, «La départementalisation nous projeta de l'univers rural post-esclavagiste aux systèmes inédits d'un pays développé » (172). C'est ce qui a accéléré le processus de dépossession.

Glissant dénonce également dans Le discours antillais toute entreprise de dépersonnalisation qui viderait l'être antillais de sa substance. C'est pourquoi, à la notion d'homme universel, il oppose celle d'homme pluriel, riche de plusieurs racines culturelles. Il reprend dans Philosophie de la relation cette thématique de la recomposition du monde. Ainsi, il en arrive à exiger une nouvelle posture dans le rapport de soi à l'autre parce que le sort des uns et des autres est inextricablement lié, comme l'explique sa théorie de la relation : «Parce que de même qu'on ne peut pas sauver une langue toute seule en laissant périr les autres, de même on ne peut pas sauver une nation ou une ethnie en laissant dépérir les autres. Et c'est ce que j'appelle la Relation » (99). Le constat est que cette interdépendance des langues et des cultures du monde entraîne incontestablement celle des identités.

Sous ce rapport, étant dans une situation de dépendance et d'aliénation dès le départ, les Antillais, certes baignés dans le multiculturalisme et la diversité raciale, ne pouvaient avoir une idée juste d'eux-mêmes, car ils se regardaient à travers les yeux de l'Autre, c'est-à-dire de ceux d'un Occidental. Qualifiant cette situation de domination silencieuse, Chamoiseau propose de la combattre afin d'arrêter l'emprise des anciens maîtres sur les Antilles en prônant un retour aux valeurs de soi. Il s'agit de mettre en avant ses propres références pour que le peuple antillais puisse 
survivre sans tomber dans l'ethnocentrisme primaire qui consiste, selon Tzvetan Todorov, à «ériger, de manière indue, les valeurs propres à la société à laquelle j'appartiens en valeurs universelles » (19). Pour Chamoiseau, toute conception des rapports humains basée sur une telle posture qui écrase les valeurs culturelles étrangères est à bannir. A contrario, la diversité culturelle née de la rencontre des divers peuples dans les îles doit être valorisée et sauvegardée pour un meilleur vivre ensemble. En effet, la mise en avant d'une seule composante culturelle ou ethnique peut créer des frictions et des incompréhensions dans la société.

La domination silencieuse est certes moins violente que celle imposée de force, mais elle est tout aussi aliénante. Difficilement perceptible, elle dépossède l'Antillais de son être et de ses moyens de défense en le rendant amnésique vis-à-vis de ses propres références. L'aliénation se fait donc en douceur et avance masquée en remplaçant une forme de domination par une autre. En fait, pour Chamoiseau, la Martinique ou la Guadeloupe, entre autres, demeurent des colonies en proie à l'étouffement de leurs diversités culturelles. Par conséquent, le combat contre l'idéologie dominante doit amener l'Être antillais à s'accepter d'abord dans ce qu'il a de spécifique et de particulier. Il s'agit pour lui de mettre en avant ses propres références culturelles et de s'opposer à la soumission culturelle et sociale prônée par la France pour imposer sa perception du monde à ceux qu'elle domine.

Dans ce contexte, toute création littéraire ou artistique se présente comme une entreprise d'émancipation culturelle et idéologique. Ainsi, la première des missions de l'écrivain sera d'abord de déclencher chez les Antillais une prise de conscience par rapport à la domination silencieuse. Le titre même de l'ouvrage de Chamoiseau à cheval entre l'essai et l'autobiographie, Écrire en pays dominé, souligne l'urgence de la situation et interpelle les écrivains quant au rôle à jouer dans cet éveil des consciences. En fait, l'abolition de l'esclavage, en éliminant les signes les plus manifestes de l'oppression, a pu donner aux Antillais le sentiment d'être libres alors que ce n'est pas le cas selon l'essayiste. Les mécanismes à l'œuvre sont plus subtils et provoquent une aliénation de l'imaginaire. Il faut donc, selon Chamoiseau, une littérature plus engagée et plus militante afin d'en faire un instrument de libération, un lieu de démarcation et d'affirmation identitaire par rapport à l'ancienne puissance coloniale. 


\section{La construction d'un contre-discours identitaire}

Prenant conscience de l'acculturation qui guette leur peuple à travers la politique d'assimilation instaurée par la départementalisation, les écrivains antillais Glissant et Chamoiseau, entre autres, ont développé des discours identitaires nouveaux afin de redéfinir les conditions d'un meilleur vivre-ensemble. Ainsi, Glissant, à travers la théorie de l'Antillanité, fait appel à toutes les composantes des Caraïbes alors que Chamoiseau questionne l'identité antillaise en prenant en compte sa diversité culturelle et ethnique.

\section{La mise en avant de l'Antillanité chez Glissant}

En mettant en avant le concept de l'Antillanité, Glissant indique de manière forte la voie à suivre afin d'appréhender dans toutes ses composantes la question identitaire aux Antilles. Conçue comme étant une démarche identitaire ouverte et plurielle, l'Antillanité lui permet de reconstituer les déchirures sociales causées par le passé colonial en s'appropriant effectivement tout l'espace. Ce positionnement discursif résulte d'une prise de conscience de la pluralité ethnique qui caractérise les îles. En effet, le souci de prendre en compte le destin particulier des îles de la Caraïbe et d'affirmer leur identité culturelle, sociale et politique a poussé certains auteurs à explorer d'autres voies que celles tracées et défendues par Césaire.

Glissant ne laissait-il pas entendre dans son discours intitulé « Le romancier noir et son people. Notes pour une conférence » prononcé au Deuxième congrès des écrivains et artistes noirs que « le propre du roman est de suivre la réalité dans ses moindres replis. [...] ; le propre du roman est de développer les mille et une variations de la réalité » (31) ? La réalité étant pour lui celle de la société antillaise dans son ensemble, Glissant pose ici les prémisses de ce qui va constituer les fondements conceptuels de sa théorie de l'Antillanité laquelle débouchera sur l'esthétique du divers, une notion développée par Victor Segalen. Cependant, Segalen définit le divers par rapport à la sensation d'exotisme « qui n'est autre que la notion du différent; la perception du Divers ; la connaissance que quelque chose n'est pas soi-même [...]»(36). Segalen entend «par esthétique l'exercice de ce même sentiment [...] » (73) à travers la pratique littéraire. Mais Glissant, lui, inscrit la notion du divers dans une démarche littéraire qui va à l'encontre de toute pratique présentant « la valeur d'un lieu comme valeur universelle » (Philosophie, 103). Ainsi, à la vision occidentale universaliste du monde, il oppose celle qui met en exergue le divers, lequel correspond à ses yeux aux «différences qui se rencontrent, s'ajustent, s'opposent, s'accordent et produisent de l'imprévisible » (98), à l'image de la société caribéenne elle-même. 
En effet, la Caraïbe est peuplée d'hommes et de femmes qui viennent de tous les continents, d'Afrique, d'Europe, d'Amérique et d'Asie. Ne pas tenir compte de ce fait, c'est occulter une partie importante de la population de ces îles, et c'est là un des reproches majeurs faits à Césaire. Dans L'intention poétique, Glissant se positionne clairement sur cette question par rapport à Césaire et affirme :

On ne s'enracine pas dans des vœux (même qui clament la racine) ni dans la terre lointaine (même si c'est la terre-mère, l'Afrique), parce qu'on recommence de la sorte un (autre) processus abstrait d'universel, là où il faut contribuer par sa richesse propre à la relation totale. Il faut marcher du vœu au réel : ce sera pour épanouir un autre vœu. Ces œuvres de Césaire prendront leur plein sens quand elles seront intégrées à une littérature du pays-même [...]. (143)

Pour Glissant, la culture antillaise telle qu'elle était véhiculée, c'est-à-dire rabaissée au rang de sous-culture, demandait à être repensée. L'esthétique du divers et la poétique de la relation relatées dans ses différents essais lui permettent de repenser les rapports entre les différentes cultures du monde. D'où la nécessité de formuler un concept nouveau, celui de l'Antillanité en l'occurrence, conçu par opposition à la Négritude césairienne. Par l'Antillanité, Glissant entend prendre en charge l'ensemble des particularismes propres aux différentes îles que compte la Caraïbe à travers une réappropriation de soi et la reconnaissance du métissage culturel. Il veut surtout souligner la richesse culturelle de cette partie du globe où toutes les civilisations du monde sont entrées en contact. C'est pourquoi, à l'identité racine unique, Glissant substitue l'identité rhizome qui est une racine démultipliée permettant à chaque identité de s'étendre dans un rapport à l'autre. Mais au préalable, il faut reconnaître et accepter le phénomène de créolisation du monde, qu'il définit ainsi dans l'Introduction à une poétique du divers : «Les phénomènes de créolisation sont des phénomènes importants, parce qu'ils permettent de pratiquer une nouvelle approche de la dimension spirituelle des humanités. Une approche qui passe par une recomposition du paysage mental de ces humanités d'aujourd'hui » (17). En fait, la créolisation est un mélange de cultures. Elle est aussi, selon Glissant, la pensée de demain où se mélangent toutes les cultures du monde. Il se sert de ce concept pour désigner tous ces émigrés, ces exilés nés de parents immigrés dans les Caraïbes. Le mélange des cultures qui ne sont pas dans des rapports hiérarchiques aboutit toujours à de nouvelles cultures : «Car la créolisation suppose que les éléments culturels mis en présence doivent obligatoirement être 'équivalents en valeur' pour que cette créolisation s'effectue réellement » (17). Cependant, selon Glissant, la créolisation dépasse largement la Caraïbe qui fait partie d'un monde éclaté. Du coup, il en appelle, pour cerner ce monde en diffraction, à une pensée 
archipélique, c'est-à-dire une pensée qui ne privilégie ni le centre, ni la périphérie. Il constate que « la pensée archipélique convient à l'allure de nos mondes. Elle en emprunte l'ambigu, le fragile, le dérivé. Elle consent à la pratique du détour, qui n'est pas fuite ni renoncement. Elle reconnaît la portée des imaginaires de la Trace, qu'elle ratifie » (31). Évoquant le détour et la trace, Glissant remarque que c'est par ces moyens que les hommes, arrachés à leurs patrimoines culturels et à leurs traditions ancestrales, ont pu reconstituer la mémoire de leur lointain passé.

Aussi lie-t-il ce formidable parcours du génie antillais à la diversité de son environnement qu'il appelle paysage. Le paysage, chez Glissant, concerne à la fois les conditions géographiques et climatiques, économiques et humaines. Ces conditions orientent, guident et symbolisent une identité en égale mesure personnelle (homme pluriel) et collective (société multiculturelle). En comparaison à d'autres paysages, le paysage antillais a pour signes distinctifs des îles en archipel composées de la mer, de l'océan et de la végétation tropicale. La culture antillaise est de ce point de vue le reflet de ce paysage insulaire qui permet de tout relativiser, à l'image de la mer Caraïbe qui « porte à l'émoi de la diversité » (15). Ainsi, pour Glissant, le monde devrait être pensé dans sa pluralité et dans sa diversité, et non pas dans l'unicité. L'Antillanité est en effet la prise en charge de cette identité plurielle qui n'est pas une absence d'identité. Pour l'essayiste, la réalité antillaise est une réalité multiculturelle qui n'a jamais connu de centre, ni de périphérie, c'est une mosaïque. Selon toujours Glissant, l'intérêt de cette mosaïque est d'intégrer plusieurs dimensions culturelles. Toutefois, de manière graduelle, on passera de l'Antillanité dans les années 1957-1958 avec Glissant à la Créolité lancée en 1989 par Chamoiseau, Confiant et Jean Bernabé afin d'ancrer leurs discours dans la réalité locale de leur terroir.

La Créolité comme reflet de la diversité culturelle

Chamoiseau met également en avant les spécificités de son terroir à travers l'esthétique de la créolité afin de pallier les conséquences désastreuses de l'assimilation culturelle et politique aux Antilles. C'est la raison pour laquelle, avec Confiant et Bernabé, ils proclament haut et fort dans L'éloge de la créolité leur ferme volonté de se déprendre, tant sur le plan culturel que littéraire, de toute «expression mimétique»(15) et entendent renouveler les démarches au niveau de la production et de la réception des œuvres.

Alors même qu'ils se réclament de Césaire comme l'un de leurs inspirateurs, à l'image de Glissant, Chamoiseau, Confiant et Bernabé ont décidé de donner une nouvelle perspective à leur littérature, en mettant de côté tout ce qui pourrait les détourner des réalités locales de la société 
antillaise en général, et martiniquaise en particulier. Ainsi prendront-ils leur distance par rapport à leurs prédécesseurs. Chamoiseau, notamment, reproche à la Négritude son incapacité à prendre en charge les véritables réalités culturelles des peuples concernés, et sa tendance « à domicilier notre identité dans une culture niée, déniée et reniée »(18). Même si, à la décharge de Césaire, ils reconnaissent la nécessité de redécouvrir, dans un premier temps, l'Histoire occultée des peuples sous domination coloniale, ils exigent en priorité la prise en compte de leur identité métisse.

Aussi prendront-ils la peine, dans L'éloge de la créolité, de se définir d'abord sur le plan identitaire : «Ni Européens, ni Africains, ni Asiatiques, nous nous proclamons Créoles. Cela sera pour nous une attitude intérieure, mieux : une vigilance, ou mieux encore, une sorte d'enveloppe mentale au mitan de laquelle se bâtira notre monde en pleine conscience du monde » (13). Ces propos illustrent leur volonté de se tourner d'abord vers eux-mêmes à travers «la vision intérieure » (15). Cette dernière est une démarche nouvelle qui permet de saisir la complexité antillaise en privilégiant la dimension créole. À propos de cette vision, Kemedjio Cilas indique dans De la négritude à la créolité. Édouard Glissant, Maryse Condé et la malédiction de la théorie : «La vision intérieure inaugure les conditions de possibilité d'une expression authentique qui passe par une exor-cisation de la vieille fatalité de l'extériorité par une méfiance vis-à-vis de tout regard extérieur dont la seule présence risque toujours d'invalider les procédés les plus justes. La vision intérieure inaugure un renversement dans le champ de la connaissance antillaise [...] » (268).

Par cette démarche d'introspection et ce positionnement identitaire, les écrivains antillais mettent sur un même pied l'ensemble des composantes culturelles de leur société. Dès lors, ils admettront qu'il y a aux Antilles une sorte de mosaïque culturelle, comme l'avait notamment constaté Glissant auparavant. En effet, pour Chamoiseau, il faut promouvoir l'imaginaire de la diversité et reconnaître chacun dans sa différence. Il dira : « une des entraves de notre créativité fut le souci obsessionnel de l'Universel. [...] D'où, à l'exception des miracles individuels, notre naufrage esthétique. Notre balbutiement » (Éloge, 50). Cet échec collectif explique la nécessité d'un nouveau départ, lequel implique la reconnaissance, sur le plan littéraire, de l'enrichissement mutuel et le rôle primordial que doit jouer l'écrivain antillais qui vit une situation particulière au niveau linguistique : «La littérature créole d'expression française aura donc pour tâche urgente d'investir et de réhabiliter l'esthétique de notre langage » (47), le langage étant pour Chamoiseau l'usage libre et créateur d'une langue quelle qu'elle soit. Selon lui, l'écrivain n'est jamais dans la 
langue communiquée ; il a un style qui naît de son aventure individuelle. Il est celui qui doit trouver sa langue dans la langue de tous, et son style naît de l'interférence culturelle.

En outre, toujours selon Chamoiseau, la langue résulte d'un mélange de cultures et constitue un carrefour qui se situe au-delà des frontières nationales. C'est pourquoi il faut prendre la langue à son compte, la moduler et l'enrichir d'apports divers : "Vivre en même temps la poétique de toutes les langues, c'est surtout rompre l'ordre coutumier de ces langues, renverser leurs significations établies. C'est cette rupture qui permettra d'amplifier l'audience d'une connaissance littéraire de nous-mêmes » (48). En fait, L'éloge de la créolité augure cette rupture épistémologique et annonce les fondements d'un discours critique nouveau que ces auteurs s'évertueront à mettre en œuvre dans leurs productions littéraires et artistiques, en partant d'un constat : « Nous sommes fondamentalement frappés d'extériorité. Cela depuis les temps de l'antan jusqu'au jour d'aujourd'hui. Nous avons vu le monde à travers le filtre des valeurs occidentales, et notre fondement s'est trouvé 'exotisé' par la vision française que nous avons dû adopter. Condition terrible que celle de percevoir son architecture intérieure, son monde, les instants de ses jours, ses valeurs propres, avec le regard de l'Autre » (14). La vision intérieure et l'acceptation de soi constituent les voies de salut pour sortir de cette situation de dépendance multiséculaire. Cette démarche nouvelle, ainsi initiée dans L'éloge de la créolité, sera poursuivie dans Écrire en pays dominé. Dans cet essai polygénérique qui emprunte à la fois au conte et au roman autobiographique, Chamoiseau préconise la libération totale et complète de l'imaginaire antillais pour sortir du système de domination qui bride tout génie créateur aux Antilles. Sur le plan formel, la diversité typographique reflète aussi cette volonté subversive dans sa pratique esthétique. Ainsi, les « Sentimenthèques » ou souvenirs littéraires de l'auteur et les «Inventaire d'une mélancolie », récits du vieux guerrier, sont écrits en petits caractères et mis en retrait dans le corps du texte et manifestent à titre illustratif cette pluralité de voix qui traversent toute l'œuvre de Chamoiseau.

\section{Conclusion}

En définitive, la départementalisation présentée comme une solution politique aux contradictions culturelles, raciales et politiques créées par des siècles d'esclavage et de colonisation aux Antilles s'est révélée être, pour les peuples d'Outre-mer, une autre forme de dépendance plus insidieuse, à savoir la dépendance administrative et bureaucratique vis-à-vis de la Métropole. Malgré la distribution d'allocations de toutes sortes et la promesse d'égalité des 
chances entre les citoyens, la départementalisation est pour l'Antillais synonyme d'acculturation et de dépossession idéologique. C'est la raison pour laquelle certains intellectuels antillais ont fait de la revendication d'une identité plurielle pouvant refléter la situation sociologique réelle de leurs îles un leitmotiv pour l'émancipation de leurs concitoyens.

Il est certes difficile pour l'écrivain antillais, habitué à être vu et à se voir à travers les yeux d'autrui, de se dégager de l'imaginaire de l'Autre, de créer ses propres références avec ses propres critères sociaux, culturels et idéologiques. Pourtant, l'essentiel de la production discursive antillaise s'articulera autour de la problématique post-coloniale suivante : comment désaliéner les mentalités après plusieurs siècles d'assimilation politique, culturelle et idéologique ? Il transparaît toutefois dans la pratique esthétique et discursive d'écrivains comme Glissant et Chamoiseau que la désaliénation de l'Antillais ne sera effective que lorsqu'il aura non seulement cessé de se percevoir au travers du prisme idéologique blanc dominant, mais également lorsqu'il échappera à l'opposition mentale et symbolique Blanc / Noir, en instaurant de nouvelles normes culturelles fondées sur la diversité raciale et l'acceptation mutuelle. À ce titre, la créolité, à travers son esthétique et sa vision du monde telle que l'expose Chamoiseau dans Écrire en pays dominé, et la théorie de l'Antillanité développée par Glissant qui s'inspire des réalités locales des archipels des Caraïbes, sont des solutions que la société antillaise doit explorer pour se retrouver. Remarquons qu'à la différence du mouvement de la Négritude, ces dernières ne prônent ni la civilisation de «l'universel», ni le renfermement sur soi, mais une ouverture au monde par la promotion du « diversel » compris comme étant la somme de toutes les différences.

\section{Bibliographie}

Bernabé, Jean, Patrick Chamoiseau et Raphaël Confiant. L'éloge de la créolité. Paris : Gallimard, 1989.

Césaire, Aimé. Le discours sur le colonialisme. Paris : Présence africaine, 1995 [1955].

Chamoiseau, Patrick. Écrire en pays dominé. Paris : Gallimard, 1997.

Cilas, Kemedjio. De la négritude à la créolité. Edouard Glissant, Maryse Condé et la malédiction de la théorie. Hamburg : Litt, 1999.

Glissant, Édouard. Philosophie de la relation. Paris : Gallimard, 2009.

---. Introduction à une poétique du divers. Paris : Gallimard, 1996 [1995].

---. La poétique de la relation. Paris : Gallimard, 1990. 
---. Le discours antillais. Paris : Seuil, 1997 [1981].

---. L’intention poétique. Poétique II. Paris : Gallimard, 1997 [1969].

---. « Le romancier noir et son people. Notes pour une conférence ». Paris : Présence africaine, $1957: 26-31$.

Segalen, Victor. Essai sur l'exotisme, une esthétique du divers. Montpellier : Fata Morgana, 1978.

Todorov, Tzvetan. Nous et les autres. La réflexion française sur la diversité humaine. Paris : Seuil, 1989.

\footnotetext{
Notes

${ }^{1}$ Le 19 mars 1946, la Loi de départementalisation est votée par le Parlement français. Cette loi, à l'initiative d'Aimé Césaire, est l'aboutissement de plusieurs années de revendications populaires aux Antilles pour une reconnaissance de l'égalité entre les «vieilles colonies» et la Métropole. Les quatre colonies (la Réunion, la Guadeloupe, la Martinique et la Guyane) deviennent des départements français.

${ }^{2}$ En tant que représentants de leur îles respectives, Léopold Bissol de la Martinique, Gaston Monnerville de la Guyane et Raymond Vergès de la Réunion étaient avec Aimé Césaire les défenseurs et signataires de la loi du 19 mars 1946 sur la départementalisation.
} 